\title{
KNOWLEDGE MANAGEMENT IN INDUSTRY CLUSTERS AS AN INDICATION OF ENTREPRENEURSHIP
}

Bogusław Bembenek ${ }^{1}$, Teresa Piecuch ${ }^{2}$

\begin{abstract}
The article emphasizes the fact that industry clusters, as organisations, composing of various subsystems through mutual cooperation, are able to successfully attain the established objectives. Their capability to work efficiently is determined, however, by the quantity and quality of the knowledge they possess.

Knowledge, as a component of intellectual capital, is currently the key strategic resource of clusters. Even more crucial is the way in which a manager of a cluster achieves, processes, transfers, and protects the knowledge. Assuming that knowledge management influences the results of a cluster, the article determines the significance of this process, and indicates the need for ceaseless development.
\end{abstract}

JEL Classification Numbers: L26, M2, O15, O33, 034, DOI: http://dx.doi.org/10.12955/cbup.v2.441

Keywords: management, resources, knowledge, cluster, partnership, entrepreneurship, development

\section{Introduction}

Functioning in a turbulent environment, strong pressure for reducing the operational expenditure and the necessity of permanent protection against all kinds of turbulences necessitates design and implementation of a knowledge management system by cluster managers. Devising in a cluster, a coherent, complex system of knowledge management is one of the toughest challenges to be faced by a cluster manager. The system entails complete, consolidated, unfragmented knowledge of processes that occur in a cluster, and their impact on the outside environment. It also involves a strategic approach to the application of communication and information technologies.

The manager of a cluster, possessing proper knowledge management in a cluster, is able to seize any given opportunity more effectively, and to build and permanently develop the resistance of this organization to the results of unfavorable events occurring in the environment. Thus, professional knowledge management in a cluster, as an indication of entrepreneurial attitude, can constitute the basis for its competitiveness potential.

The purpose of this article is to determine the role of knowledge management in the process of managing the development of a cluster. The consideration, which was undertaken on the basis of the selected results of theoretical and empirical research, aims at emphasizing the need for successful acquisition and the use of knowledge in a cluster.

\section{Concept of industry cluster - unity in diversity}

Porter (1990) claims that clustering is a dynamic process, and as one competitive firm grows, it creates a demand for other related industries. As the cluster develops, it becomes a mutually reinforcing system where benefits flow back and forth throughout the industries within the cluster. Owing to numerous advantages resulting from the development of clustering concept, clusters are presently treated not only as a pro-competitive, pro-developing, and pro-innovative phenomenon, but also as a successful tool for building national and regional innovation system, which is understood as an integrated network of enterprises and R\&D institutions basing on an effective mechanism of knowledge generation and diffusion.

According to the economic and management literature, industry clusters are groups of firms in the same location, comprising a production system with spillovers that can be vertical and/or horizontal (Strojer-Madsen, Smith, \& Dilling-Hansen, 2002). Industry clusters are defined as geographic

\footnotetext{
${ }^{1}$ Bogusław Bembenek, Rzeszow University of Technology, Poland, bogdanb@prz.edu.pl

${ }^{2}$ Teresa Piecuch, Rzeszow University of Technology, Poland, tpiecuch@ prz.edu.pl
} 
concentration of: interconnected firms in a particular field with links to related institutions; companies in a specialized field that cooperate with local community to efficiently share resources leading to improved economic gains and equitable enhancement of environmental quality (Zhihua Zeng, 2011; Anbumozhi, 2007). Barkley \& Henry (2001) believes that industry clusters include groupings of firms with diverse characteristics, and, as a result, varied potentials for employment growth and local economic development. It is also arguable that industry cluster is a geographically bounded collection of similar and/or related firms that together create competitive advantages for member firms and the local economy (Barkley \& Henry, 2005). The cluster model emphasizes internal linkages, whereby cluster gains are furthered by local firm cooperation, local institutions, and local social capital (Nadvi \& Barrientos, 2004). Industry clusters differ significantly with respect to characteristics of the strategy, dominant sectors, extent of interdependencies among firms, availability of govern-mental and institutional support, and employment generation potentials (Barkley \& Henry, 2001). Romanelli \& Khessina (2005) shows that regional industrial identities are grounded in the configuration of industry clusters that populate a region. Markusen (1994) argues that shared characteristics among industry clusters permit them to be grouped into four general types (Markusen, 1994; Paytas, Gradeck, \& Andrews, 2004; Portugal Ferreira, Ribeiro Serra, Kramer Costa, Maccari, \& Ritor Couto, 2012):

- Marshallian clusters (networked cluster, industrial districts): comprised primarily of locally owned, small and medium-sized businesses concentrated in craft-based, high technology, or producer services industries. Firms in a networked cluster enjoy advantages not available to firms elsewhere, including access to local knowledge and labor markets, low transportation and transaction costs, cultures of flexibility, trust, cooperation, and available local infrastructure supporting specialized sales, service, and supplier networks.

- hub and spoke - are dominated by one or several large firms surrounded by smaller suppliers and related activities. Smaller firms in the region can be closely linked to the dominant firm through supply chains, or may simply be located nearby to take advantage of the benefits of agglomeration.

- satellite platforms - are industry clusters dominated by large and almost completely externally oriented firms. These firms have minimal intra-cluster exchanges in favor of stronger ties to the parent headquarters and other sister subsidiaries elsewhere.

- state-anchored clusters (institutional clusters): are regions where the local business structure is dominated by a public or non-profit entity (e.g. military base, university, or government offices). Institutional clusters are generally externally focused, responding first to their goals and needs of the institution, which may be externally focused and only secondarily providing benefits to the local economy.

Rosenfeld (1997) suggested that industry cluster is a geographically bounded concentration of similar, related or complementary businesses, with active channels for business transactions, communications and dialogue that share specialized infrastructure, labor markets and services; and that it is faced with common opportunities and threats. Firms in an industry cluster may interact through purchase-sale relationships; inter-firm cooperation in product development, marketing, or research; or a shared reliance on specialized services and labor markets (Barkley \& Henry, 2005).

Industry cluster can help overcome the two kinds of market failure on R\&D. Firstly, firms in clusters can more easily build collaborative networks. Secondly, when spillover is high, collaborative R\&D with rival firms internalizes knowledge spillovers and enhances the incentive to invest in $R \& D$ (Nishimura \& Okamuro, 2010).

The most obvious examples are benefits, which arise from the easy access firms in the same industry clusters enjoy (Albu, 1997), including: 
- specialized local suppliers of inputs and services;

- a local mobile pool of labor with highly relevant skills and knowledge;

- a local industrial atmosphere in which relevant technological know-how and ideas are "in the air" and are readily available to all.

Peneder (1999) explains that the industry cluster concept normally implies more than literal meaning of density by reference to a hypothesis, which states that the geographic agglomeration of economic activity may lead to improved technological or economic performance of the participating units. Basing on a holistic approach to a cluster, it can be assumed that a cluster is a category of a system, i.e. an organized collection of elements (tangible and intangible components), maintaining mutual, diverse, direct and indirect relations that creates a qualitatively new whole, which is distinctive in the environment. Moreover, clusters are purpose-oriented systems, whose orderliness consists of particular parts contributing to the success of the whole. Clusters as created by humans, artificial systems, possess a characteristic attribute of natural systems, i.e. they strive for survival and development. In regards to the clusters, there are two strategic ways for their development, namely: internal development — basing on the strategic potential they possess, and external development - basing on tangible and intangible resources, which are possessed by external stakeholders. Function and the development of a cluster are, however, strictly determined by the quality of integration of the structure members representing the business, scientific, and social environment, which is measured with their readiness to cooperate for the benefit of the achievement of common objectives. The cluster, as a developing organisation, not only can it constitute an example of an organisation in motion, but also an organisation based on knowledge, which is learning, or is intelligent.

Industry clusters have certain institutional ability to learn constantly and share pieces of advice, technical solutions, and information concerning new technologies, good practices, market trends, and also great innovative abilities and reciprocal supplementing. This kind of a cluster is frequently identified with an organisation basing not only on the individual knowledge, which is possessed by particular members of this structure, but also on the collective knowledge developed jointly during the realisation of various ventures - including inter- and intra-organisational interactions. The power of the knowledge-based clusters consists of the ability to design, create, develop and transfer the knowledge within this structure, and the ability to integrate different research fields, around specific technological solutions, or organisational learning. In order to permanently secure their competitiveness, cluster structures should not only concentrate on managing the already possessed knowledge, but also identify still new scopes of knowledge, properly attain process, and transfer them in internal and external environments, while simultaneously protecting them.

\section{Knowledge as the basis the intellectual capital of the cluster}

Knowledge as an intangible resource of particular features is a result of its organisation, location, application, effectiveness, and formal and informal character of individual experiences of people, who create and use it, constitute presently the main source of growth and competitive advantage. Particularly, in conditions of the development of knowledge-based industry and information society, it is emphasized that knowledge is strongly related to the human factor. Simon (1991) considered that the knowledge is created by individual human being; and to be efficient in knowledge creation and storage, individuals need to become specialized. Knowledge as an organizational resource of cluster is a fluid mix of framed experience, values, contextual information, and expert insight that provides a framework for evaluating and incorporating new experiences and information (Baskerville \& Dulipovici, 2006). Analyzing the characteristics of knowledge and its different dimensions, it can be assumed that knowledge is created as a result of: social interactions between individuals or organizations, a process of learning, performance of certain tasks, and identification and resolution of 
the assumed problems. The general knowledge of a cluster is, to a considerable extent, the knowledge of particular members of the cluster. In order to enhance its productivity, the manager of a cluster should coordinate it appropriately. Effective transformation of knowledge from the individual to the organizational level is essential for knowledge to become the basis of organizational capability (Kogut $\&$ Zander, 1993). Certainly, during the process of reciprocal teaching in a cluster, common knowledge is created. The ability of a cluster to generate new, common knowledge indirectly influences its development. This role of common knowledge is one among a broader set of issues concerning knowledge integration in the development and production of goods and services in a cluster (Grant \& Baden-Fuller, 2004).

Explicit knowledge and tacit knowledge, occurring in a cluster, constitute the core component of intellectual capital of the cluster. Explicit knowledge, as formal, externalized, and systematized knowledge, is easy to transfer by means of wildly available, common forms of transmission. Whereas, tacit knowledge, as silent knowledge, is transferred mainly verbally in face-to-face contact; and it is gathered as the experience, competencies, and intuition increases. The scopes of the explicit knowledge and tacit knowledge in a cluster can be derived from: firstly, individual knowledge of particular members of a cluster and so-called "knowledge workers," employed in its organisations; secondly, knowledge recorded in documents, databases, and procedures; thirdly, knowledge legally protected by patents, licences, trademarks, copyrights, formulas, and trade secrets.

Owing to the diversity of some approaches to identification and measurement of intellectual capital, still, there is a lack of a uniform standard in defining it. Intellectual capital is commonly defined as the (Kong \& Thomson, 2009):

- a sum of an organization's resources encompassing collective tacit knowledge, human skills, creativity, experience and any intellectual resource that can contribute to value creation for the organization;

- collective knowledge that is embedded in the personnel, organizational routines and network relationships of an organization.

Intellectual capital characterises the value of a cluster, determining its operational and strategic efficiency in acting and functioning on the market. The value created on the basis of intellectual capital results from reciprocal interactions between particular elements of human, structural, and relational capital (Rehman et al., 2011), wherein:

- human capital is experience, expertise, skill and creativity of employees, which can be further encouraged by investing more in their training programs;

- $\quad$ structural capital is all systems, procedures, databases, copy rights, structural procedures, rules and policies, which are important for decision-making;

- relational capital is all the relationship of organization with different stakeholders, cumulative trust, experience and knowledge on building relationship, or partnership.

Steward (1997) emphasizes that intellectual capital is formed mainly by the knowledge, which is useful to the organization. It develops the characteristics of the capital only when one can process and use it for the purpose of the organizational development. A key method, serving the development of the intellectual capital, is efficient (successful and effective) knowledge management in a cluster.

\section{Importance of knowledge management in a cluster}

Knowledge development in a cluster is a dynamic phenomenon, since it comes as a result of an interaction between people and organizations; it can assume various dimensions, e.g. practical, theoretical, explicit, tacit, cognitive, automatic, expertise, individual, collective, hypothetic, and 
interdisciplinary knowledge. Furthermore, the knowledge in a cluster can be acquired through (Tidd, J., Bessant, J., \& Pavitt, 2005): mobilization of tacit knowledge, collaborative formal and informal research and development work, reverse engineering, competitive intelligence, contracting research and development work outside the cluster, strategic research and development partnership (consortia and joint-venture), patents and licenses purchasing, e-learning, coaching, mentoring, building project teams, and brainstorming.

Valuable knowledge of a cluster, considered the most strategic resource of a cluster, finds expression in innovative products, processes, technologies, and organizations. The knowledge of a cluster, basing on the acquired information, experience, intuition, consciousness and understanding, is interpreted as all the knowledge and skills used by the decision-makers, including cluster members to solve problems; such knowledge can comprise of the knowledge on the level of a cluster as a whole (organisation), a project team, or a cluster member. Managing cluster knowledge, understood in such a way, refers to the process of building a cluster "based on knowledge and opened to knowledge," which integrates, among others individuals, strategy, organizational culture, strategic potential of cluster members to use the knowledge effectively.

Knowledge management, in theory and practice, has been recognized as an important process that organizations need to develop in order to gain sustainable competitive advantages. This process can be understood as (Bhojaraju, 2005; Ajiferuke, 2003):

- a complex, systematic process of: gathering, managing and sharing of employees' knowledge capital throughout the organization, and finding, selecting, organizing, distilling and presenting information, which improves an employee's comprehension in a specific area of interest;

- a collection of dynamic processes that governs the creation, dissemination, and utilization of knowledge in an organization.

Knowledge management is the management technique used in cluster development, which aims to increase the capability of companies to learn and gain knowledge through cooperation, and is a kind of discipline that helps spread knowledge of individuals or groups across organizations in such ways that directly affect performance (Sureephong, Chakpitak, Ouzrout, Neubert, \& Bouras, 2007). Managing the knowledge as a strategic resource of a cluster regards boosting of knowledge efficacy through ceaseless identification, organisation and formation of its development processes. The process of knowledge management in a cluster is strongly influenced by individual and collective intelligence, complex network of cooperation, organizational culture, and the potential of a cluster. Petrides \& Nodine (2003) showed that knowledge management brings together three core organizational resources, people, processes, and technologies, to enable the organization to use and share information more effectively. Analyzing the processes of knowledge management in a cluster, in the context of business processes, four typical sub-processes can be distinguished (Sarvay, 1999):

- organizational learning, in which a cluster attains and develops its knowledge;

- knowledge production, which is linked to an active transfer of data and information into knowledge, making it useful in solving the emerging problems;

- knowledge distribution, where a cluster provides the access to common knowledge to its members and other key stakeholders, and enables them to take advantage of it;

- knowledge protection, aiming at protecting knowledge from its loss, unauthorised use, among others, through improper selection, preservation, and updating.

Complex approach toward identification, acquisition, development and protection of knowledge indicates that knowledge management is based on the processes of organisational learning, and 
constitutes a designed system, which should support the manager in making decisions, attaining, analysing, and using the new and already existing knowledge. A cluster manager, being responsible for managing the knowledge in the cluster, should demonstrate the ability and skill of identifying, characterizing, and removing certain barriers for knowledge development. Certainly, it will be easier for them to diagnose and reduce technical and organisational barriers, rather than psychosocial and cultural ones. A crucial feature in this case is uncertainty, concerning the way of using the collective knowledge by the staff managing the cluster, and also particular cluster members, including the competitors. In addition, the inner intentions of the partners within the scope of cooperation determine the implementation of knowledge management, to the extent of which they are honest, as a sign of an opportunistic attitude.

Basant (2002) argues that sector specialization of a cluster is likely to be important for knowledge generation and diffusion in two ways:

- technological opportunities differ across sectors. Besides, potential for intra-sectoral spillovers and learning possibilities may also be different for different sectors;

- technological opportunities emerging from inter-sectoral linkages can be significant for certain sectors enhancing the potential for inter-sectoral technology spillovers.

Access to knowledge resources in geographical proximity depends on an active involvement in knowledge development and exchange networks or skilled labor markets (Stahlecker, 2012). According to Cowan, David, \& Foray (2000), cluster structures are gradually developing their own language of communication and information interpretation, which enhances the ability of the cluster members to coordinate their activities, share knowledge or even non-verbalised, tacit knowledge. In terms of knowledge sharing, clusters are collaborative modes of business practice that enhance competitiveness because the knowledge sharing network upgrades skills and knowledge more quickly (Cooke, 2002).

The research ${ }^{3}$, which involved Polish clusters in 2010, indicated that cluster members exchange commercial and technological data, information about contractors or clients, and also support each other in acquisition, and transfer of information concerning the legal changes, possibilities of cooperation, including the draft and the fulfilment of common projects. Certainly, it is not a standard practice. Knowledge exchange is characterized mainly for the clusters with a high level of social capital, including confidence capital. The research demonstrates that the exchange of the commercial information is more popular in the case of clusters, which possess bigger number of participants receiving external financial appropriations, and the ones whose members are oriented toward real cooperation — not only toward personal benefits.

The research ${ }^{4}$ conducted in Poland in 2012 on 35 clusters proved that the presence of active scientific institutions, as well as R\&D institutions in a cluster, is conducive to the knowledge transfer. The

\footnotetext{
3 "Cluster benchmarking in Poland" project was performed in the period from March to November 2010 and comprised the comparative analysis of 47 operating clusters. It was performed within the framework of the initiative launched by the Polish Agency for Enterprise Development titled: "Development of Human Resources Through the Promotion of Knowledge, Transfer and Dissemination of Innovation" (Cluster Benchmarking, 2010).

${ }^{4}$ Benchmarking studies were performed within the framework of the initiative launched by the Polish Agency for Enterprise Development titled "Cluster Benchmarking in Poland - 2012" in the period from June to November 2012. The applied method of studies allowed for gaining the position of particular clusters on the basis of the entire population and identification of their position in relation to the benchmark. 35 clusters were studied. Analyzing their strengths, it has been noticed that on the map of clusters in Poland, there are more and more, whose share of investments on R\&D in expenditures on innovative activity in the core of the cluster within the last two years, has exceeded $25 \%$. Within the last two years, more than five research projects have been
} 
results regarding the creation of knowledge and innovation in clusters functioning according to the Dutch model, which characterises itself with an active participation of the science sector in the cluster work, were $26 \%$ better than the results of the other clusters. One in three clusters declared informal exchange of knowledge and information amidst their members. Opportunities for knowledge transfer can take the forms of: regular meetings of the members, get-togethers, joint formulation and implementation of the project concepts, joint scientific reports and analyses, and publications. An important and noticeable trend in clusters operation in Poland is the development of communication platforms, dedicated to particular needs of the cluster members. These platforms very frequently possess sophisticated tools, making the cooperation between the members easier (e.g. within the scope of projects management, joint invoicing, and e-learning). During the course of the research, informal contact and cooperation within informal knowledge exchange were considered to be the key advantage of Polish clusters.

\section{Conclusion}

Decision makers in cluster structures are beginning to perceive the need for effective knowledge management. They are becoming more and more convinced that state of the art management system would enable successful and effective knowledge management, especially on the level of choosing strategy and undertaking strategic operations. The ability to manage the knowledge in a cluster is presently an important attribute, conducive to building and developing the market potential, which enhances the decision-making process, design and implementation of various changes, in order to ensure the stability of functionality and development of a cluster in turbulent conditions of the global market environment. In the knowledge-based clusters, one can observe a simplified information flow, developed processes of learning, which stimulates innovation; there is a higher consciousness among the members, also among the cluster's manager and the leader, concerning the role of knowledge in building strategic advantage. Sustaining multi-dimensional balance of a cluster in the process of knowledge development is a result of the established intra- and inter-organisational relations, which enable: more effective identification of partners for the future projects, shortened learning cycle, and permanent improvement of this structure.

Informal exchange of knowledge in a cluster is conducive to better comprehension of the mutual needs of the cluster's members, as well as of the external key stakeholders. Systematic acquisition of the knowledge from the environment can also serve to redefine the established development strategy, generate of new values for the cluster, and maintain flexibility in adapting to the dynamically changing needs of the market. However, before the decision is made to transform a cluster into a knowledge factory, the following issues must be considered (Perechuda, 2010):

- the advancement of strategic thinking and actions on cluster,

- the level of social capital, including trust capital,

- the vision of development a knowledge in cluster,

- the philosophy of transforming a cluster in a knowledge factory,

- the knowledge gap in the cluster,

- the mission of knowledge sharing,

- the existing knowledge potential,

- the desired potential of a cluster,

implemented; the members of the cluster and products of the cluster were at least on 10 foreign markets (HołubIwan, 2012). 
- cold and hot knowledge in a cluster,

- the responsibility of organization structure and management system for acquiring, creating and sharing knowledge,

- human resources potential with regard to their transformation into knowledge workers,

- knowledge-creating oriented culture of the cluster,

- the information-communication system which facilitates the sharing of knowledge.

In the process of knowledge management, it is significant to skilfully utilize state-of-the-art communication and information technologies, e.g. technologies assisting in localizing and codifying explicit knowledge, technologies of gathering explicit knowledge, technologies supporting extraction of new knowledge from the resources of explicit knowledge, technologies of learning, and technologies of integrating and making knowledge accessible in an integrated way. Obviously, the intranet and internet networks play a crucial role in enabling effective communication inside and outside the cluster; and thus they are conducive to the knowledge transfer, and reusability by various stakeholders.

The approach toward knowledge management in a cluster, being strongly influenced by people managing this kind of structure, can attest the entrepreneurial attitude. The cluster itself is considered to be a structure that has come as a result of entrepreneurial initiatives of individuals and organizations orientated toward fulfilling a common objective. It is commonly regarded as an environment conducive to the creation and implementation of various technological solutions, innovations (concerned a vital tool in entrepreneurs' hands), especially, in knowledge-based high-tech clusters. Creation of the environment, favorable to the entrepreneurship development in a cluster, involves the state of the art, continuous development, creative behavior in the process of its acquisition, organisation, and management. As a result of the knowledge, a change in the approach of clusters managers, and other persons engaged in its functioning along with the entrepreneurs who created this structure toward the environment, from the attitude-oriented mindset toward objective adjustment by fulfilling its requirements and doing what it expected - assuming active creation, co-creation, and even precedence to the changes and tendencies occurring in it. A manager of a knowledge-based cluster can be called an intellectual entrepreneur, because in this case the knowledge constitutes strategic resources for them. Moreover, it is important that the scopes of the knowledge, gathered for the benefit of the structure members, fulfil their role; serve the progress, development, and useful changes; and reinforce the competitiveness of a cluster in the environment. Such a manager will take care of the accessibility of knowledge and its transfer between the interested subjects, absorption of knowledge, or will skilfully use the informative feedback. It significantly enables shortening and simplification of the decision-making processes. As a result, of effective knowledge management, a cluster can be characterized with an entrepreneurial structure, hence, it reacts quicker to the changes of the environment and effectively takes advantage of them.

Consequently, it can be claimed that the knowledge is the basis for the functionality of industry cluster, which conditions its development; whereas, entrepreneurial management of this resource is one of the tools enabling a cluster manager to satisfy s trategic objectives, seize opportunities, and build the competitive potential more efficiently even in complex conditions of the environment. Moreover, the significance of knowledge management in the process of cluster development increases systematically as the content of this structure expands, under the influence of the dynamic changes of the environment, as a result of the emergence of new challenges in the modern world. 


\section{References}

Ajiferuke, I. (2003). Role of information professionals in knowledge management programs. Informing Science Journal, 6 , 247.

Albu, M. (1997). Technological Learning and Innovation in Industrial Clusters in the South. Electronic Working Papers Series, Paper no. 7. Science Policy Research Unit, University of Sussex.

Anbumozhi, V. (2007). Eco-Industrial Clusters in Urban-Rural Fringe Areas. Hyogo: Institute for Global Environmental Strategies.

Barkley, D., \& Henry, M. (2001). Advantages and Disadvantages of Targeting Industry Clusters. Clemson, South Carolina: Clemson University Public Service Activities.

Barkley, D., \& Henry, M. (2005). Targeting Industry Clusters for Regional Economic Development., Clemson, South Carolina: Clemson University.

Basant, R. (2002, January). Knowledge Flows and Industrial Clusters an Analytical Review of Literature. Ahmedabad: Indian Institute of Management.

Baskerville, R., \& Dulipovici, A. (2006). The Theoretical Foundations of Knowledge Management. Knowledge Management Research \& Practice, 4, 84.

Bhojaraju, G. (2005). Knowledge Management: Why Do We Need It for Corporate. Malaysian Journal of Library \& Information Science, 10(2), 37-38.

Cluster Benchmarking in Poland - 2010. Survey Report. (2010). Warszawa: PARP.

Cooke, P. (2002). Knowledge Economies: Clusters, Learning and Co-Operative Advantage. Routledge, London, New York.

Cowan, R., David, P.A., Foray, D. (2000). The Explicit of Economics of Knowledge Codification and Tacitness. Industrial and Corporate Change, 9(2), 211-253.

Grant, R.M., \& Baden-Fuller, Ch. (2004, January). A Knowledge Accessing Theory of Strategic Alliances. Journal of Management Studies, 41(1), 79.

Hołub-Iwan, J. (2012). Cluster Benchmarking in Poland - 2012. General Report. Warszawa: PARP.

Kogut, B., \& Zander, U. (1993). Knowledge of the Firm and the Evolutionary Theory of the Multinational Corporation. Journal of International Business Studies, 24(4), 625-646.

Kong, E., \& Thomson, S.B. (2009). An Intellectual Capital Perspective of Human Resource Strategies and Practices. Knowledge Management Research \& Practice, 7, 356, 358.

Markusen, A. (1994). Sticky Places in Slippery Space: The Political Economy of Postwar Fast-Growth Regions. Working Paper, no. 79. New Brunswick: Center for Urban Policy Research.

Nadvi, K., \& Barrientos, S. (2004). Industrial Clusters and Poverty Reduction. Vienna: United Nations Industrial Development Organization.

Nishimura, J., \& Okamuro, H. (2010). R\&D Productivity and the Organization of Cluster Policy: An Empirical Evaluation of the Industrial Cluster Project in Japan. DRUID Working Paper, no. 10-06. Danish Research Unit for Industrial Dynamics.

Paytas, J., Gradeck, R., \& Andrews, L. (2004). Universities and the Development of Industry Clusters. Pittsburgh: Economic Development Administration U.S. Department of Commerce.

Peneder, M. (1999). Creating a Coherent Design for Cluster Analysis and Related Policies: The Austrian TIP Experience. OECD, Boosting Innovation: The Cluster Approach. Paris.

Perechuda, K. (2010). Knowledge Diffusion Methods in a Networking Company. Wroclaw: Publishing House of Wroclaw University of Economics.

Petrides, L., \& Nodine, T. (2003). Knowledge Management in Education: Defining the Landscape. Half Moon Bay, CA: The Institute for the Study of Knowledge Management in Education.

Porter, M. (1990). The Competitive Advantage of Nations. New York: Free Press.

Portugal Ferreira, M., Ribeiro Serra, F., Kramer Costa, B., Maccari, E.A., \& Ritor Couto, H. (2012). Impact of the Types of Clusters on the Innovation Output and the Appropriation of Rents from Innovation. Journal of Technology Management \& Innovation, 7(4), 75.

Rehman, W., Rehman, Ch. A., Rehman, H., \& Zahid, A. (2011). Intellectual Capital Performance and Its Impact on Corporate Performance: An Empirical Evidence from Modaraba Sector of Pakistan. Australian Journal of Business and Management Research, 1(5), 9. 
Romanelli, E., \& Khessina, O.M. (2005). Regional Industrial Identity: Cluster Configurations and Economic Development. Organization Science, 16(4), 345.

Rosenfeld, S. (1997). Bringing Business Clusters into the Mainstream of Economic Development. European Planning Studies, 5, 3-23.

Sarvay, M. (1999). Knowledge Management and Competition in the Consulting Industry. California Management Review, 41(2), 96.

Simon, H.A. (1991). Bounded Rationality and Organizational Learning. Organization Science, 2, 125-134.

Stahlecker, T., Kroll, H., \& Baier, E. (2012). Identification of knowledge-driven clusters in the EU. Brussels: European Commission.

Steward, T.A. (1997). Intellectual Capital. New York: Bantam Doubleday Dell Publishing Group.

Strojer-Madsen, E., Smith, V., \& Dilling-Hansen, M. (2002). Industrial Clusters, Firm Location and Productivity - Some Empirical Evidence for Danish Firms. Working Paper 03-26. Department of Economics, Aarhus School of Business.

Sureephong, P., Chakpitak, N., Ouzrout, Y., Neubert, G., \& Bouras, A. (2007). Knowledge Management System Architecture for the Industry Cluster. Industrial Engineering and Engineering Management. IEEE International Conference, 1971.

Tidd, J., Bessant, J., \& Pavitt, K. (2005). Managing Innovation, Integrating Technological, Market and Organizational Change. Chichester: John Wiley \& Sons, Ltd.

Zhihua Zeng, D. (2011). How Do Special Economic Zones and Industrial Clusters Drive China's Rapid Development? Policy Research Working Paper, no. 5583. The World Bank Africa Region Finance \& Private Sectors Development. 